\title{
A high accuracy approximation for half - space problems with anisotropic scattering
}

\author{
M. H. Haggag ${ }^{1,2}$, H.F. Mashali ${ }^{2}$ \\ ${ }^{1}$ (Physics Department, Faculty of Science, Taif University, Taif, K.S.A) \\ ${ }_{2}^{2}$ (Physics Department, Faculty of Science, Mansoura University, Mansoura, Egypt)
}

\begin{abstract}
An approximate model, which is developed previously, is extended to solve the half-space problems in the case of extremely anisotropic scattering kernels. The scattering kernel is assumed to be a combination of isotropic plus a forward and backward leak. The transport equation is transformed into an equivalent fictitious one involving only multiple isotropic scattering, therefore permitting the application of the previously developed method for treating isotropic scattering. It has been shown that the method solves the albedo half - space problem in a concise manner and leads to fast converging numerical results as shown in the Tables. For pure scattering and weakly absorbing medium the computations can be performed by hand with a pocket calculator Keywords: Approximate model, Anisotropic scattering, Albedo problem, Half-space, Transport equation.
\end{abstract}

\section{Introduction}

The study of radiative transfer and neutron transport in anisotropically scattering media usually develops the scattering function in a series of Legendre polynomials of the scattering angle [1-6]. This is useful when the number of terms needed is small. However, when the scattering has some strongly preferred directions, many terms must be used and the method will be cumbersome. There are some scattering functions for which a development in Legendre polynomials is not useful because of much stronger anisotropy may exist. In an attempt to overcome this problem, that is to estimate the effect of strong anisotropy on the solution ,a special scattering model which combines backward scattering and forward scattering with an admixture of isotropic scattering in arbitrary proportions has been used [7-13] . In the case of purely forward scattering with an isotropic component there are no particular problems in casting the transport equation into a suitable form for solution. On the other hand, when backward scattering is included it is necessary to employ a transformation due to Inönü [14] to cast the equation into an equation corresponding to isotropic scattering.

In the present paper, we wish to generalize a method previously introduce to demonstrate the computational merits of it when applied to the anisotropic scattering model mentioned above. This method is a semi-analytical approximate method and suitable for obtaining highly accurate solutions for one dimensional transport problems. It was introduced by the authors [15-17] for treating multiple isotropic scattering in a homogeneous half - space problems.

Now let us consider the one - speed, time independent, homogeneous transport equation,

$$
\mu \frac{\partial}{\partial Z} \psi(\mathrm{z}, \mu)+\psi(\mathrm{z}, \mu)=\omega_{0} \int_{-1}^{1} \Pi\left(\mu^{\prime} \rightarrow \mu\right) \psi\left(\mathrm{z}, \mu^{\prime}\right) \mathrm{d} \mu^{\prime}
$$

where $\boldsymbol{\psi}$ is the angular intensity, with distance measured in units of mean free path, $\mu$ is the direction cosine of the angle between the positive z-axis and the particle velocity vector, and $\boldsymbol{\omega}_{\mathbf{0}}$ is the single scattering albedo (mean number of secondaries per collision). Here $\boldsymbol{\Pi}\left(\boldsymbol{\mu}^{\prime} \rightarrow \boldsymbol{\mu}\right)$ represents the scattering law, which we write as [7],

$$
\Pi\left(\mu^{\prime}, \mu\right)=\ell \delta\left(\mu^{\prime}+\mu\right)+m \delta\left(\mu^{\prime}-\mu\right)+\frac{1}{2} n
$$

where $\boldsymbol{\ell}$ and $\mathrm{m}$ are real constants in the range $\mathbf{0} \leq \boldsymbol{\ell}, \boldsymbol{m} \leq \mathbf{1}$ and give the fraction of particles which emerge from a collision in the backward and forward directions, respectively. In addition $\boldsymbol{n}=\mathbf{1}-\boldsymbol{\ell}-\boldsymbol{m}$ and gives the fraction of particles which emerge isotropically from a collision. Using equation (2) we can write equation (1) as

$$
\mu \frac{\partial}{\partial z} \psi(z, \mu)+\left(1-m \omega_{o}\right) \psi(z, \mu)=\ell \omega_{o} \psi(z,-\mu)+\frac{1}{2} n \omega_{o} \int_{-1}^{1} \psi\left(z, \mu^{\prime}\right) d \mu^{\prime}
$$

In terms of the reduced optical variable 
equation (3) becomes

$$
\tau=\left(\mathbf{1}-\mathbf{m} \boldsymbol{\omega}_{\mathbf{o}}\right) \mathbf{z}
$$

$$
\mu \frac{\partial}{\partial \tau} \psi(\tau, \mu)+\psi(\tau, \mu)=\alpha \psi(\tau,-\mu)+\frac{1}{2} \beta \int_{-1}^{1} \psi\left(\tau, \mu^{\prime}\right) d \mu^{\prime}
$$

where we have defined

and

$$
\alpha=\frac{\ell \omega_{0}}{1-m \omega_{0}}
$$

$$
\beta=\frac{n \omega_{0}}{1-m \omega_{0}}
$$

Equation (5) shows that forward scattering $(\boldsymbol{\ell}=\mathbf{0})$ does not introduce any analytical complications since the form of the resulting equation of transport is no different from the usual equation. However, backward scattering does lead to an equation of transport considerably different from the usual one, because the arguments $\pm \boldsymbol{\mu}$ cause it to be a type of integro-differential functional equation.

\section{The Albedo Problem}

The albedo problem is the problem of obtaining the angular radiation or neutron density everywhere in a source free half - space, if a prescribed angular density is incident on the surface $z=0$. Now, we seek solutions of the homogeneous transport equation (5) subject to the boundary conditions

$$
\boldsymbol{\psi}((0, \mu)=F(\mu), \quad \mu>0
$$

and that

$$
\boldsymbol{\psi}(\boldsymbol{\tau}, \boldsymbol{\mu}) \rightarrow \mathbf{0} \quad \text { as } \quad \tau \rightarrow \infty
$$

As discussed by Inönü, [14], we can transform the problem for anisotropic scattering into an equivalent equation with an isotropic scattering. Equation (5) reduces to the form valid for the purely isotropic scattering,

where

$$
\mu \frac{\partial}{\partial x} \Psi(x, \mu)+\Psi(x, \mu)=\frac{\omega}{2} \int_{-1}^{1} \Psi\left(x, \mu^{\prime}\right) d \mu^{\prime}
$$

$$
\begin{array}{r}
x=\tau \sqrt{1-\alpha^{2}} \\
\omega=\beta /(1-\alpha)
\end{array}
$$

The relation between angular distributions of (5) and (9) is

$\boldsymbol{\psi}(\boldsymbol{\tau}, \boldsymbol{\mu})=\frac{B+1}{2} \Psi(\mathrm{x}, \mu)-\frac{B-1}{2} \Psi(\mathrm{x},-\mu)$

with

$$
\mathrm{B}=[(1-\alpha) /(1+\alpha)]^{1 / 2}
$$

Now, if $\Psi(\mathrm{x}, \mu)$ is a solution of (9) with $\boldsymbol{\omega}$ given by (11), then $\boldsymbol{\psi}(\tau, \mu)$, represented by (12) and (13), is a solution of (5). It is now straightforward to translate the boundary condition (8a) on $\boldsymbol{\psi}$ into equivalent one on $\boldsymbol{\Psi}$. We find

$$
\Psi(\mathbf{0}, \boldsymbol{\mu})=(\mathbf{1}-\boldsymbol{R}) \boldsymbol{F}(\boldsymbol{\mu})+\boldsymbol{R} \Psi(\mathbf{0},-\boldsymbol{\mu}) \quad \boldsymbol{\mu}>0,
$$


where $\mathrm{R}$ is given by

$$
R=(B-1) /(B+1)
$$

The problem is thus reduced to that of solving (9) complemented by the reflected boundary condition (14) at the surface $\mathrm{x}=0$ of the half - space.

\section{Analysis And Method Of Solution}

We now want to transform (9) into an integral equation suitable for numerical treatment. For this purpose we introduce the total intensity $\boldsymbol{\Phi}(\mathrm{x})$ associated with the angular intensity $\boldsymbol{\Psi}(\mathrm{x}, \mu)$ appearing in (9). Now equations (9) and (14) can be formally solved, $\boldsymbol{\mu}>0$, to give,

$$
\begin{gathered}
\Psi(\mathrm{x}, \mu)=\frac{\omega}{2 \mu} \int_{0}^{\mathrm{x}} \mathrm{e}^{-\frac{1}{\mu}(\mathrm{x}-\mathrm{y})} \Phi(\mathrm{y}) \mathrm{dy}+\frac{\omega \mathrm{R}}{2 \mu} \int_{0}^{\infty} \mathrm{e}^{-\frac{1}{\mu}(\mathrm{x}+\mathrm{y})} \Phi(\mathrm{y}) \mathrm{dy}+(1-\mathrm{R}) \mathrm{F}(\mu) \mathrm{e}^{-\frac{\mathrm{x}}{\mu}}, \\
\Psi(\mathrm{x},-\mu)=\frac{\omega}{2 \mu} \int_{\mathrm{x}}^{\infty} \mathrm{e}^{-\frac{1}{\mu}(\mathrm{y}-\mathrm{x})} \Phi(\mathrm{y}) \mathrm{dy}
\end{gathered}
$$

Integrating (16) and (17) over $\mu$ to obtain the following inhomogeneous integral equation for $\boldsymbol{\Phi}(\mathrm{x})$,

$$
\Phi(\mathrm{x})=\mathrm{S}(\mathrm{x})+\frac{\omega}{2} \int_{0}^{\infty} \boldsymbol{H}(\mathrm{x}, \mathrm{y}) \Phi(\mathrm{y}) \mathrm{dy}
$$

where

$$
\mathrm{H}(\mathrm{x}, \mathrm{y})=\boldsymbol{E}_{1}(|\mathrm{x}-\mathrm{y}|)+\boldsymbol{R} \boldsymbol{E}_{1}(\mathrm{x}+\boldsymbol{y}),
$$

with $\boldsymbol{E}_{n}(\mathrm{x})$ denoting the exponential integral function of order $\mathrm{n}$ and the inhomogeneous term $\mathrm{S}(\mathrm{x})$ is defined by

$$
\mathrm{S}(\mathrm{x})=(1-\mathrm{R}) \int_{0}^{1} F(\boldsymbol{\mu}) \mathrm{e}^{-\mathrm{x} / \mu} d \boldsymbol{\mu}
$$

To completely determine the angular distributions $\Psi(\mathrm{x}, \pm \mu)$ as given by (16) and (17) we must solve Eq. (18)

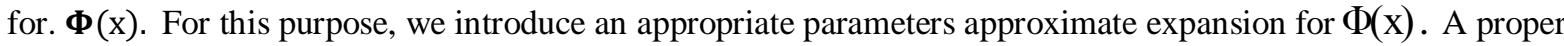
choice for the parametric expansion is of the form

$$
\Phi(\mathrm{x})=\mathrm{Ae}^{-x / v}+\sum_{n=0}^{N} A_{n} E_{n+2}(\mathrm{x})
$$

where $\mathrm{A}$ and $\mathrm{A}_{\mathrm{n}}$ are expansion coefficients to be determined and $v$ is the positive root of

$$
1=\frac{\omega v}{2} \ln \left[\frac{1+v}{1-v}\right]
$$

Using this expansion, the angular distributions everywhere in the half -space can be calculated from, $\mu>0$,

$$
\begin{aligned}
\Psi(\mathrm{x}, \mu)= & (1-\mathrm{R}) \mathrm{F}(\mu) \mathrm{e}^{-\mathrm{x} / \mu}+\frac{\omega v}{2}\left[\frac{\mathrm{e}^{-\mathrm{x} / \nu}-\mathrm{e}^{-\mathrm{x} / \mu}}{\nu-\mu}+\mathrm{R} \frac{\mathrm{e}^{-\mathrm{x} / \mu}}{v+\mu}\right] \boldsymbol{A}+ \\
& \frac{\omega}{2 \boldsymbol{\mu}} \mathrm{e}^{-\mathrm{x} / \boldsymbol{\mu}} \sum_{\boldsymbol{n}=\mathbf{0}}^{\mathrm{N}}\left[\eta_{\boldsymbol{n}+2}(\mathrm{x}, \mu)+\mathrm{R} \eta_{\boldsymbol{n}+2}(\infty,-\mu)\right] \boldsymbol{A}_{\boldsymbol{n}}
\end{aligned}
$$

and

$$
\Psi(\mathrm{x},-\mu)=\frac{\omega v}{2} \frac{\mathrm{e}^{-\mathrm{x} / v}}{v+\mu} \boldsymbol{A}+\frac{\omega}{2 \mu} \mathrm{e}^{\mathrm{x} / \boldsymbol{\mu}} \sum_{n=0}^{\mathrm{N}}\left[\eta_{n+2}(\infty,-\mu)-\eta_{n+2}(\mathrm{x},-\mu)\right] \boldsymbol{A}_{\boldsymbol{n}}
$$

The functions $\boldsymbol{\eta}_{\mathbf{n}}(\mathrm{x}, \mu)$ are defined by

$$
\boldsymbol{\eta}_{\boldsymbol{n}}(\mathrm{x}, \mu)=\int_{0}^{\mathrm{x}} \mathrm{e}^{\mathrm{y} / \mu} \mathrm{E}_{\boldsymbol{n}}(\mathrm{y}) \mathrm{dy}
$$


and explicitly are given in [15 ]. Specializing (23) to get

$$
\Psi(0,-\boldsymbol{\mu})=\frac{\omega v}{2} \frac{\mathrm{A}}{\boldsymbol{v}+\boldsymbol{\mu}}+\frac{\omega}{2 \mu} \sum_{\mathrm{n}=0}^{\mathrm{N}} \boldsymbol{\eta}_{\mathrm{n}+2}(\infty,-\mu) \mathrm{A}_{\boldsymbol{n}}
$$

and from (12), the exit angular distribution of the original problem is given by

$$
\boldsymbol{\psi}(\mathbf{0},-\boldsymbol{\mu})=-\boldsymbol{R} \boldsymbol{F}(\boldsymbol{\mu})+(\mathbf{1}+\boldsymbol{R}) \Psi(\mathbf{0},-\boldsymbol{\mu})
$$

Another quantity, of physical interest, relevant to the present problem is the albedo which is defined by

$$
\mathrm{A}^{*}=\frac{\int_{0}^{1} \mu \psi(0,-\mu) d \mu}{\int_{0}^{1} \mu F(\mu) d \mu}
$$

Using Eq. (26) , A* can be expressed as

$\mathrm{A}^{*}=-R+\frac{\omega}{2}(1+R)\left[\int_{0}^{1} \mu F(\mu) d \mu\right]^{-1}\left\{\left[v-v^{2} \ln \left(1+\frac{1}{v}\right)\right] A+\sum_{n=0}^{N} \mathrm{~J}_{2, n+2} A_{n}\right\}$,

where the constants $\mathrm{J}_{\mathrm{n}, \mathrm{m}}$ are integrals evaluated to yield

$(\mathbf{n}+\mathbf{m}-1) \mathbf{J}_{\mathbf{n}, \mathbf{m}}=(-1)^{\mathrm{n}+1}\left[\ln 2+\sum_{\mathrm{k}=1}^{\mathrm{n}-1}(-1)^{\mathrm{k}} / \mathbf{k}\right]+(-1)^{\mathrm{m}+1}\left[\ln 2+\sum_{\mathrm{k}=1}^{\mathrm{m}-1}(-1)^{\mathrm{k}} / \mathbf{k}\right]$

\section{The EXPANSION COEFFICIENTS}

To solve for the expansion coefficients $A$ and $A_{n}$ we substitute (21a) into (18) then, operate on the resulting expression first with the operator $\int_{0}^{\infty} \mathrm{e}^{-\frac{\mathrm{x}}{v}} \ldots . \boldsymbol{d x}$, and second with $\int_{0}^{\infty} \boldsymbol{E}_{\mathrm{m}+2}(\mathrm{x}) \ldots \ldots . . \mathrm{dx}$ where $\mathrm{m}=$ $0,1,2, \ldots, N$. As a result of these two operations, one obtains $(\mathrm{N}+2)$ linear algebraic equations for $(\mathrm{N}+2)$ unknown expansion coefficients which are written as

$$
\mathrm{T}(v) \mathrm{A}+\sum_{\mathrm{n}=0}^{\mathrm{N}} \mathrm{T}_{\boldsymbol{n}}(v) \mathrm{A}_{\mathrm{n}}=\mathrm{d}(v)
$$

and

$$
\mathrm{T}_{\mathrm{m}}(v) \mathrm{A}+\sum_{\mathrm{n}=0}^{\mathrm{N}} \mathrm{D}_{\mathrm{mn}} \mathrm{A}_{\boldsymbol{n}}=\mathrm{d}_{\boldsymbol{m}}
$$

The analytic expressions for the integrals of $T, T_{n}$ and $D_{m n}$ are evaluated to give

$$
\begin{aligned}
& T(v)=\left\{\begin{array}{cl}
\frac{v}{2}\left[1-\omega v(1+R) \ln \left(\frac{v+1}{v}\right)+\omega R \frac{v}{v+1}\right], & 0<\omega<1 \\
\frac{1}{4}(1-R), & \omega=1
\end{array}\right. \\
& \mathrm{T}_{n}(v)=\left\{\begin{array}{cc}
\frac{\omega v}{2}\left[Y_{n+2}^{-}(v)+R Y_{n+2}^{+}(v)-(1+R) \mathrm{J}_{1, n+2}\right], & 0<\omega<1 \\
\frac{1}{2}(1-R) \mathrm{J}_{2, n+2}, & \omega=1
\end{array}\right.
\end{aligned}
$$

and for $\mathrm{D}_{\mathrm{mn}}$ one has

$$
\mathrm{D}_{\mathrm{mn}}=\mathrm{J}_{\boldsymbol{m}+2, \boldsymbol{n}+2}-\frac{\boldsymbol{\omega}}{2} \mathrm{H}_{\mathrm{m}+2, \mathrm{n}+2}
$$

where

$$
\begin{aligned}
& (-1)^{\mathrm{m}+\mathrm{n}} \mathrm{H}_{\mathrm{mn}}=\left[\mathrm{R}+(-1)^{\mathrm{n}+1}+(-1)^{\mathrm{m}+1}\right] \widetilde{\boldsymbol{\psi}}_{m+n+1}+\left[R+(-1)^{m+1}\right] \sum_{i=1}^{n-1} \frac{(-1)^{i}}{i} \boldsymbol{\psi}_{m+n+1-i}+ \\
& {\left[\mathrm{R}+(-\mathbf{1})^{\mathrm{n}+\mathbf{1}}\right] \sum_{i=\mathbf{1}}^{m-1} \frac{(-1)^{i}}{i} \boldsymbol{\psi}_{m+n+1-i}+R \sum_{i=\mathbf{1}}^{m-1} \quad \sum_{j=1}^{n-1}(-\mathbf{1})^{i+\mathrm{j}} \frac{1}{i \mathrm{j}(\mathrm{m}+\mathrm{n}-\mathrm{i}-\mathrm{j})}}
\end{aligned}
$$


In (33) the numerical values of $\mathrm{Y}_{\mathrm{m}}^{ \pm}$can be calculated from the recurrence relation

$$
Y_{m}^{ \pm}(v)= \pm v\left[Y_{m-1}^{ \pm}(v)+\frac{1}{m-1} \ln \left(1 \pm \frac{1}{v}\right)-\mathrm{J}_{1, m-1}\right], \quad \mathbf{m}>1
$$

Where the values corresponding to $\mathrm{m}=1$ are given by :

$$
Y_{1}^{+}=v\left[\frac{1}{2} \ln ^{2}\left(1+\frac{1}{v}\right)+\frac{\pi^{2}}{12}+\sum_{n=1}^{\infty} \frac{(-1)^{n}}{n^{2}}\left(\frac{v-1}{v+1}\right)^{n}\right]
$$

and

$$
\mathrm{Y}_{1}^{-}(v)=\mathrm{Y}_{1}^{+}(v)-v \ln \left(1+\frac{1}{v}\right) \ln \left(1-\frac{1}{v}\right)
$$

In equation (35) $\psi_{\mathrm{n}}=\mathbf{J}_{1, \mathrm{n}-1}$ and $\widetilde{\psi}_{\mathrm{n}}$ are calculated from the expression

$$
\widetilde{\Psi}_{\mathrm{n}+1}=\frac{2}{\mathrm{n}} \ln ^{2} 2+\frac{2 \ln 2}{\mathrm{n}(\mathrm{n}-1)}+\frac{2(-1)^{\mathrm{n}}}{\mathrm{n}(\mathrm{n}-1)}\left[\ln 2+\sum_{\mathrm{i}=1}^{\mathrm{n}-2} \frac{(-1)^{\mathrm{i}}}{\mathrm{i}}\right]-\frac{\mathrm{n}-1}{\mathrm{n}} \widetilde{\Psi}_{\mathrm{n}} \quad, \mathrm{n}>1
$$

starting from

$$
\widetilde{\boldsymbol{\psi}}_{2}=2 \ln ^{2} 2+\frac{\pi^{2}}{6}
$$

\section{Numerical Results}

In order to illustrate the application of the foregoing analysis, we considered two cases for $\boldsymbol{F}(\boldsymbol{\mu})$.In the first case $\boldsymbol{F}(\boldsymbol{\mu})=\mathbf{1}$ whereas in the second $\boldsymbol{F}(\boldsymbol{\mu})=\boldsymbol{\delta}\left(\boldsymbol{\mu}-\boldsymbol{\mu}_{0}\right)$. In these two cases the expressions of $\boldsymbol{d}(\boldsymbol{v})$ and $\mathrm{d}_{\mathrm{m}}$, in the algebraic system of equations, respectively, are ,

$$
\begin{aligned}
d(v) & =\left\{\begin{array}{cc}
v(1-R)[1-v \ln (1+1 / v] & , \omega<1, \\
\frac{1}{2}(1-R), & \omega=1
\end{array}\right. \\
\text { and } d_{m} & =(1-R) J_{2, m+2}, \\
d(v) & =(1-R) \frac{v \mu_{0}}{v+\mu_{0}} \\
d_{m}(v) & =(1-R) \eta_{m+2}\left(0,-\mu_{0}\right)
\end{aligned}
$$

The physical aspects of the above cases are well documented, so we focus our attention on showing the convergence of the proposed method of solution. Some quantities of physical interest are calculated and compared with other available results. The calculated quantities are the medium albedo and the exist angular intensity at $\mathrm{x}=0$ for various combinations of scattering parameters $\boldsymbol{\ell}, \boldsymbol{m}, \boldsymbol{n}$ and single scattering albedo $\boldsymbol{\omega}_{\mathbf{0}}$. We keep all parameters except one fixed, and let the free parameter vary among the set of values under consideration. The numerical results are presented in Tables 1-6. From these calculations, it is seen that the numerical results obtained by the present method are converging even in the lowest order approximations. As physically expected, we observe a decrease of $\mathrm{A}^{*}$ if the strength of the forward scattering increases. Similarly the value of $\mathrm{A}^{*}$ increases if we enlarge the backward scattering part. 
Table 1.The albedo $A^{*}$ as calculated using the zero order approximation when $F(\mu)=1$ (a) present results

(b) results of [7]

\begin{tabular}{|c|c|c|c|c|c|c|}
\hline \multirow[t]{2}{*}{$(\ell, \mathrm{m}, \mathrm{n})$} & \multicolumn{2}{|c|}{$\omega_{0}=0.5$} & \multicolumn{2}{|c|}{$\omega_{0}=0.7$} & \multicolumn{2}{|c|}{$\omega_{0}=0.9$} \\
\hline & $\mathrm{a}$ & $\mathrm{b}$ & $\mathrm{a}$ & $\mathrm{b}$ & $\mathrm{a}$ & $\mathrm{b}$ \\
\hline$\left(0, \frac{2}{3}, \frac{1}{3}\right)$ & 0.05985 & 0.05985 & 0.12118 & 0.12118 & 0.29528 & 0.29528 \\
\hline$\left(\mathrm{O}, \frac{1}{3}, \frac{2}{3}\right)$ & 0.10733 & 0.10733 & 0.19946 & 0.19945 & 0.40982 & 0.40983 \\
\hline$(\mathrm{O}, \mathrm{O}, \mathbf{1})$ & 0.14654 & 0.14654 & 0.25656 & 0.25656 & 0.47802 & 0.47802 \\
\hline$\left(\frac{1}{3}, \frac{1}{3}, \frac{1}{3}\right)$ & 0.15988 & 0.15988 & 0.27183 & 0.27183 & 0.48759 & 0.48759 \\
\hline$\left(\frac{1}{3}, 0, \frac{2}{3}\right)$ & 0.18950 & 0.18951 & 0.31029 & 0.31029 & 0.52966 & 0.52966 \\
\hline \multirow[t]{2}{*}{$\left(\frac{2}{3}, 0, \frac{1}{3}\right)$} & 0.22904 & 0.22904 & 0.35794 & 0.35795 & 0.57177 & 0.57177 \\
\hline & \multicolumn{2}{|c|}{$\omega_{0}=0.95$} & \multicolumn{2}{|c|}{$\omega_{0}=0.99$} & \multicolumn{2}{|c|}{$\omega_{0}=0.999$} \\
\hline$\left(0, \frac{2}{3}, \frac{1}{3}\right)$ & 0.41896 & 0.41896 & 0.67379 & 0.67380 & 0.88164 & 0.88164 \\
\hline$\left(0, \frac{1}{3}, \frac{2}{3}\right)$ & 0.53391 & 0.53391 & 0.75507 & 0.75508 & 0.91466 & 0.91466 \\
\hline$(\mathrm{O}, \mathrm{O}, 1)$ & 0.59666 & 0.59667 & 0.79456 & 0.79456 & 0.92971 & 0.92971 \\
\hline$\left(\frac{1}{3}, \frac{1}{3}, \frac{1}{3}\right)$ & 0.60212 & 0.60212 & 0.79558 & 0.79558 & 0.92978 & 0.92978 \\
\hline$\left(\frac{1}{3}, 0, \frac{2}{3}\right)$ & 0.64047 & 0.64047 & 0.81959 & 0.81959 & 0.93884 & 0.93884 \\
\hline$\left(\frac{2}{3}, O, \frac{1}{3}\right)$ & 0.67440 & 0.67440 & 0.83751 & 0.83751 & 0.94514 & 0.94514 \\
\hline
\end{tabular}

Table 2. Shows the convergence of $A^{*}$ for various values of $\omega_{0}$ when $F(\mu)=1$

$(\ell, \mathrm{m}, \mathrm{n}) \quad \omega_{0}=0.5$

\begin{tabular}{|c|c|c|c|c|c|c|c|}
\hline \multirow{2}{*}{\multicolumn{2}{|c|}{$\mathrm{N}$}} & & & & & & \\
\hline & & 0 & 1 & 2 & 4 & 6 & [7] \\
\hline$\left(0, \frac{2}{3}, \frac{1}{3}\right)$ & & 0.059846 & 0.059848 & 0.059848 & 0.059848 & 0.059848 & 0.05985 \\
\hline$\left(\mathrm{O}, \frac{1}{3}, \frac{2}{3}\right)$ & & 0.107333 & 0.107335 & 0.107335 & 0.107335 & 0.107335 & 0.10733 \\
\hline$(\mathrm{O}, \mathrm{O}, 1)$ & & 0.146544 & 0.146544 & 0.146544 & 0.146544 & 0.146544 & 0.14654 \\
\hline$\left(\frac{1}{3}, \frac{1}{3}, \frac{1}{3}\right)$ & & 0.159882 & 0.159884 & 0.159884 & 0.159884 & 0.159884 & 0.15988 \\
\hline$\left(\frac{1}{3}, O, \frac{2}{3}\right)$ & & 0.189504 & 0.189506 & 0.189506 & 0.189506 & 0.189506 & 0.18951 \\
\hline$\left(\frac{2}{3}, 0, \frac{1}{3}\right)$ & & 0.229037 & 0.229039 & 0.229039 & 0.229039 & 0.229039 & 0.22904 \\
\hline \multicolumn{8}{|c|}{$\omega_{0}=0.7$} \\
\hline$\left(0, \frac{2}{3}, \frac{1}{3}\right)$ & & 0.121183 & 0.121184 & 0.121184 & 0.121184 & 0.121184 & 0.12118 \\
\hline
\end{tabular}


A high accuracy approximation for half - space problems with anisotropic scattering

$\begin{array}{lllllll}\left(\mathrm{O}, \frac{1}{3}, \frac{2}{3}\right) & 0.199459 & 0.199459 & 0.199459 & 0.199459 & 0.199459 & 0.19945 \\ (\mathrm{O}, \mathrm{O}, \mathbf{1}) & 0.256555 & 0.256557 & 0.256557 & 0.256557 & 0.256557 & 0.25656 \\ \left(\frac{1}{3}, \frac{1}{3}, \frac{1}{3}\right) & 0.271827 & 0.271828 & 0.271828 & 0.271828 & 0.271828 & 0.27183 \\ \left(\frac{1}{3}, \mathrm{O}, \frac{2}{3}\right) & 0.310287 & 0.310287 & 0.310287 & 0.310287 & 0.310287 & 0.31029 \\ \left(\frac{2}{3}, \mathrm{O}, \frac{1}{3}\right) & 0.357944 & 0.357945 & 0.357946 & 0.357946 & 0.357946 & 0.35795\end{array}$

$\omega_{0}=0.9$

\begin{tabular}{lllllll}
\hline$\left(\mathrm{O}, \frac{2}{3}, \frac{1}{3}\right)$ & 0.295277 & 0.295279 & 0.295279 & 0.295279 & 0.295279 & 0.29528 \\
$\left(\mathrm{O}, \frac{2}{3}, \frac{1}{3}\right)$ & 0.409820 & 0.409826 & 0.409826 & 0.409826 & 0.409820 & 0.40983 \\
$(\mathrm{O}, \mathrm{O}, 1)$ & 0.478019 & 0.478024 & 0.478024 & 0.478024 & 0.478024 & 0.47802 \\
$\left(\frac{1}{3}, \frac{1}{3}, \frac{1}{3}\right)$ & 0.487592 & 0.487593 & 0.487593 & 0.487593 & 0.487593 & 0.48759 \\
$\left(\frac{1}{3}, \mathrm{O}, \frac{2}{3}\right)$ & 0.529658 & 0.529662 & 0.529662 & 0.529662 & 0.529662 & 0.52966 \\
$\left(\frac{2}{3}, \mathrm{O}, \frac{1}{3}\right)$ & 0.571773 & 0.571774 & 0.571774 & 0.571774 & 0.571774 & 0.57177 \\
\hline
\end{tabular}

Table 3. The albedo $A^{*}$ as calculated using the zero order approximation when $\mathrm{F}(\mu)=\delta(\mu-1)$.

(a) present results

(b) results of [2]

\begin{tabular}{|c|c|c|c|c|c|c|}
\hline \multirow[b]{2}{*}{$(\ell, \mathrm{m}, \mathrm{n})$} & \multicolumn{2}{|c|}{$\omega_{0}=0.5$} & \multicolumn{2}{|l|}{$\omega_{0}=0.7$} & \multicolumn{2}{|l|}{$\omega_{0}=0.9$} \\
\hline & $\mathrm{a}$ & $\mathrm{b}$ & $\mathrm{a}$ & $\mathrm{b}$ & $a$ & b \\
\hline$\left(0, \frac{2}{3}, \frac{1}{3}\right)$ & 0.04578 & 0.04578 & 0.09454 & 0.09453 & 0.024296 & 0.024299 \\
\hline$\left(0, \frac{1}{3}, \frac{2}{3}\right)$ & 0.08337 & 0.08336 & 0.15943 & 0.15943 & 0.34878 & 0.34883 \\
\hline$(0,0,1)$ & 0.11524 & 0.11523 & 0.20866 & 0.20868 & 0.41489 & 0.41495 \\
\hline$\left(\frac{1}{3}, \frac{1}{3}, \frac{1}{3}\right)$ & 0.14605 & & 0.24636 & & 0.44126 & \\
\hline$\left(\frac{1}{3}, 0, \frac{2}{3}\right)$ & 0.16594 & & 0.27181 & & 0.47381 & \\
\hline$\left(\frac{2}{3}, 0, \frac{1}{3}\right)$ & 0.21554 & & 0.33374 & & 0.52975 & \\
\hline & $\omega_{0}=0.95$ & & $\omega_{0}=0.99$ & & $\omega_{0}=0.999$ & \\
\hline$\left(0, \frac{2}{3}, \frac{1}{3}\right)$ & 0.35750 & 0.35756 & 0.61773 & 0.61778 & 0.85476 & \\
\hline$\left(0, \frac{1}{3}, \frac{2}{3}\right)$ & 0.47081 & 0.49087 & 0.70772 & 0.70777 & 0.89451 & \\
\hline$(0,0,1)$ & 0.53548 & 0.53554 & 0.75269 & 0.75384 & 0.91283 & \\
\hline$\left(\frac{1}{3}, \frac{1}{3}, \frac{1}{3}\right)$ & 0.55010 & & 0.75163 & & 0.90977 & \\
\hline$\left(\frac{1}{3}, 0, \frac{2}{3}\right)$ & 0.58421 & & 0.77905 & & 0.92204 & \\
\hline$\left(\frac{2}{3}, 0, \frac{1}{3}\right)$ & 0.62818 & & 0.79959 & & 0.92818 & \\
\hline
\end{tabular}


A high accuracy approximation for half - space problems with anisotropic scattering

Table 4. Shows the convergence of $A^{*}$ for various value of $\omega_{0}$ when $F(\mu)=\delta(\mu-1)$

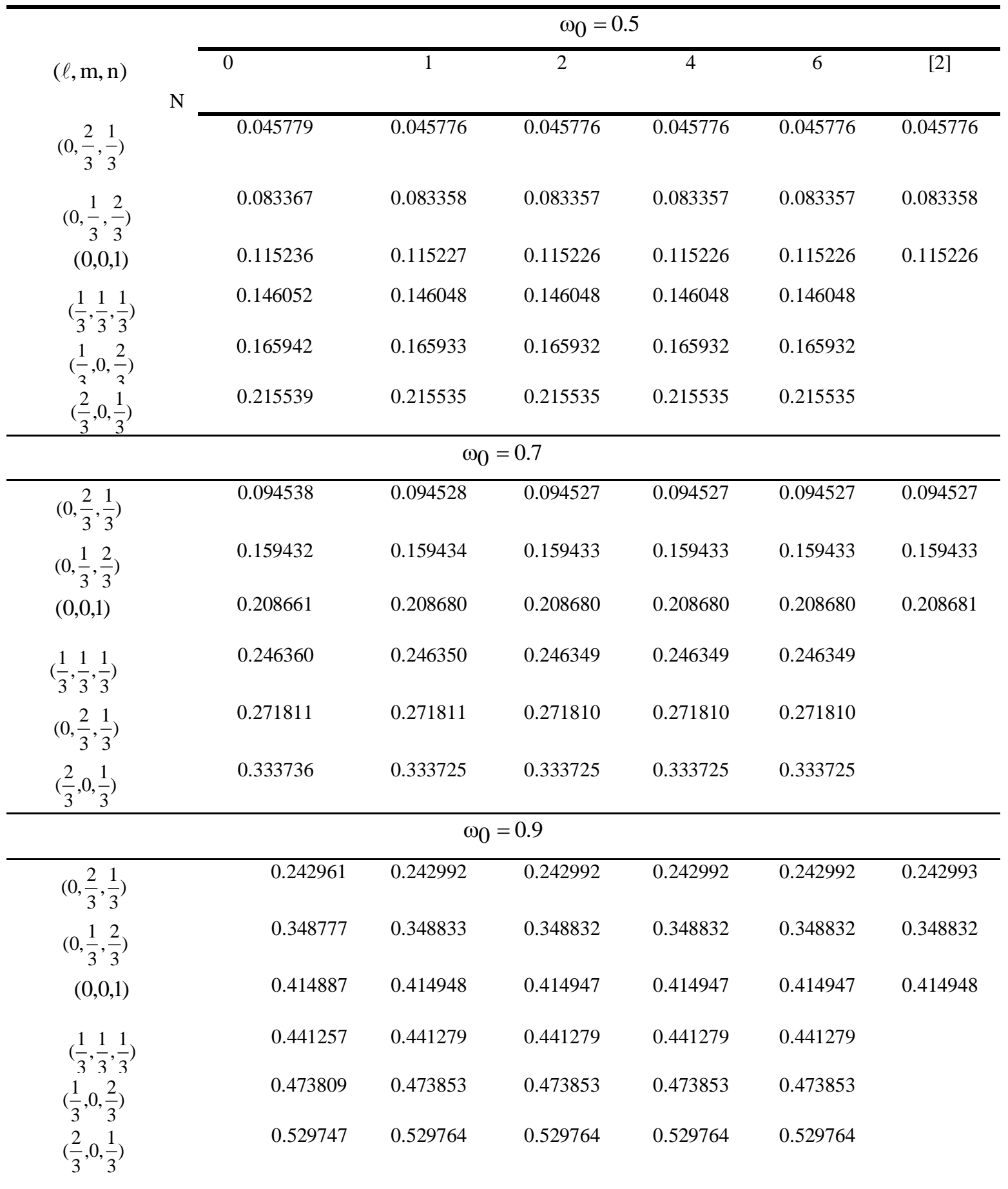

Table 5. Shows the convergence of $\psi(0,-\mu)$ at $\omega_{0}=0.9$ when $\mathrm{F}(\mu)=1$

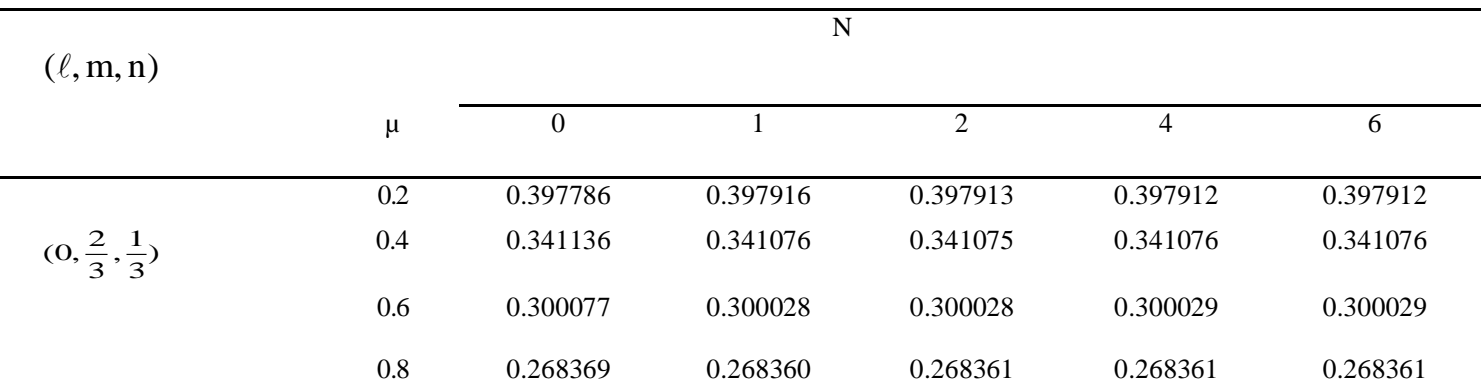


A high accuracy approximation for half - space problems with anisotropic scattering

\begin{tabular}{|c|c|c|c|c|c|c|}
\hline & 1.0 & 0.242961 & 0.242992 & 0.242992 & 0.242992 & 0.242992 \\
\hline \multirow{5}{*}{$\left(0, \frac{1}{3}, \frac{2}{3}\right)$} & 0.2 & 0.522974 & 0.523205 & 0.523204 & 0.523203 & 0.523203 \\
\hline & 0.4 & 0.462896 & 0.462801 & 0.462801 & 0.462801 & 0.462801 \\
\hline & 0.6 & 0.416760 & 0.416677 & 0.416678 & 0.416678 & 0.416678 \\
\hline & 0.8 & 0.379582 & 0.379569 & 0.379569 & 0.379569 & 0.379569 \\
\hline & 1.0 & 0.348777 & 0.348832 & 0.348832 & 0.348832 & 0.348832 \\
\hline \multirow{5}{*}{$\left(\frac{1}{3}, \frac{1}{3}, \frac{1}{3}\right)$} & 0.2 & 0.578206 & 0.578297 & 0.578296 & 0.578295 & 0.578295 \\
\hline & 0.4 & 0.528208 & 0.528165 & 0.528165 & 0.528165 & 0.528165 \\
\hline & 0.6 & 0.491882 & 0.491847 & 0.491847 & 0.491847 & 0.491847 \\
\hline & 0.8 & 0.463789 & 0.463783 & 0.463783 & 0.463783 & 0.463783 \\
\hline & 1.0 & 0.441257 & 0.441279 & 0.441279 & 0.441279 & 0.441279 \\
\hline \multirow{5}{*}{$\left(\frac{1}{3}, 0, \frac{2}{3}\right)$} & 0.2 & 0.633006 & 0.633192 & 0.633192 & 0.633191 & 0.633191 \\
\hline & 0.4 & 0.578216 & 0.578137 & 0.578137 & 0.578137 & 0.578137 \\
\hline & 0.6 & 0.536047 & 0.535979 & 0.535979 & 0.535979 & 0.555825 \\
\hline & 0.8 & 0.502023 & 0.502012 & 0.502012 & 0.502012 & 0.502012 \\
\hline & 1.0 & 0.473809 & 0.473853 & 0.473853 & 0.473854 & 0.473854 \\
\hline \multirow{5}{*}{$\left(\frac{2}{3}, 0, \frac{1}{3}\right)$} & 0.2 & 0.653884 & 0.653956 & 0.653956 & 0.653955 & 0.653955 \\
\hline & 0.4 & 0.608612 & 0.608577 & 0.608577 & 0.608577 & 0.608577 \\
\hline & 0.6 & 0.575680 & 0.575652 & 0.575652 & 0.575652 & 0.575652 \\
\hline & 0.8 & 0.550196 & 0.550191 & 0.550191 & 0.550191 & 0.550191 \\
\hline & 1.0 & 0.529747 & 0.529764 & 0.529764 & 0.529764 & 0.529764 \\
\hline
\end{tabular}

Table 6. Shows the convergence of $\psi(0,-\mu)$ at $\omega_{0}=0.9$ when $F(\mu)=\delta(\mu-1)$.

\begin{tabular}{ccccccc}
\hline & & \multicolumn{5}{c}{$\mathrm{N}$} \\
\cline { 3 - 7 }$(\ell, \mathrm{m}, \mathrm{n})$ & $\mu$ & 0 & 1 & 2 & 4 & 6 \\
\hline & 0.2 & 0.566118 & 0.569132 & 0.569796 & 0.569731 & 0.569732 \\
$\left(0, \frac{2}{3}, \frac{1}{3}\right)$ & 0.4 & 0.535748 & 0.534280 & 0.534425 & 0.534440 & 0.534440 \\
& 0.6 & 0.498014 & 0.496818 & 0.496762 & 0.496766 & 0.496766 \\
& 0.8 & 0.461789 & 0.461593 & 0.461551 & 0.461547 & 0.461547 \\
& 1.0 & 0.428975 & 0.429737 & 0.429792 & 0.429796 & 0.429796 \\
& & & & & & \\
& 0.2 & 0.771046 & 0.775664 & 0.776272 & 0.776186 & 0.776188 \\
& 0.4 & 0.751259 & 0.749362 & 0.749566 & 0.749586 & 0.749585 \\
& 0.6 & 0.713912 & 0.712266 & 0.712196 & 0.712201 & 0.712201 \\
& 0.8 & 0.673679 & 0.673404 & 0.673346 & 0.673341 & 0.673341 \\
& 1.0 & 0.634845 & 0.635949 & 0.636024 & 0.636029 & 0.636029 \\
\hline & & & & & & \\
\hline & 0.2 & 0.497888 & 0.500854 & 0.501316 & 0.501254 & 0.501254 \\
& 0.4 & 0.474896 & 0.473489 & 0.473627 & 0.473642 & 0.473642 \\
& 0.6 & 0.443241 & 0.442101 & 0.442048 & 0.442051 & 0.442051 \\
& 0.8 & 0.412040 & 0.411848 & 0.411808 & 0.411804 & 0.411804 \\
& 1.0 & 0.608577 & 0.609291 & 0.609342 & 0.609346 & 0.609346
\end{tabular}




\begin{tabular}{lcccccc} 
& 0.2 & 0.700792 & 0.705223 & 0.705819 & 0.705735 & 0.705736 \\
$\left(\frac{1}{3}, 0, \frac{2}{3}\right)$ & 0.4 & 0.687634 & 0.685766 & 0.685965 & 0.685984 & 0.685984 \\
& 0.6 & 0.655842 & 0.654229 & 0.654160 & 0.654165 & 0.654165 \\
& 0.8 & 0.620298 & 0.620021 & 0.619965 & 0.619960 & 0.619960 \\
& 1.0 & 0.739000 & 0.740066 & 0.740139 & 0.740143 & 0.740143 \\
$\left(\frac{2}{3}, 0, \frac{1}{3}\right)$ & 0.2 & 0.450062 & 0.452813 & 0.453246 & 0.453188 & 0.453188 \\
& 0.4 & 0.430906 & 0.429582 & 0.429712 & 0.429726 & 0.429726 \\
& 0.6 & 0.402962 & 0.401892 & 0.401842 & 0.401845 & 0.401845 \\
& 0.8 & 0.357046 & 0.374864 & 0.374826 & 0.374823 & 0.374823 \\
& 1.0 & 0.682625 & 0.683290 & 0.683338 & 0.683341 & 0.683341 \\
\hline
\end{tabular}

\section{Conclusion}

An efficient analytical approximation is proposed to solve radiative heat transfer or neutron transport problems in plane parallel semi - infinite media in the presence of multiple synthetic scattering kernel.The unknown function in the integral form of the transport equation is approximated by a set of trial functions with unknown coefficients. The unknown coefficients in the trial functions are found to be solutions for a system of linear, inhomogeneous algebraic equations. These coefficients are used to get numerical results of the exit distribution $\boldsymbol{\psi}(\mathbf{0},-\boldsymbol{\mu})$ and the albedo $\mathrm{A}^{*}$ from equations (26) and (28). As is seen from the Tables, the present numerical results are found to be in excellent agreement with the available results reported in the literatures

\section{References}

[1] K. M. Case and P. F. Zweifel, Linear transport theory (Addison - Wesley, Reading, Mass., 1967)

[2] S. Chandrasekhar, Radiative transfer (Dover, New York, 1960)

[3] G. I. Bell and S. Glasstone, Nuclear reactor theory (New York: Van Nostrand Reinhold, 1972)

[4] G. Spiga, F. Santarelli and C. Stramigioli, Radiative transfer in an absorbing and anisotropically scattering slab with a reflecting boundary, Int. J. Heat Mass Transfer, 23(6), 1980, 841- 852.

[5] M. Benassi, R. M. Cotta and C. E. Siewert, The $\mathrm{P}_{\mathrm{N}}$ method for radiative transfer problems with reflective boundary conditions, J. Quant. Spectrosc. Radiat. Transfer, 30(6), 1983, 547-553.

[6] L. B. Barichello, R. D. M. Garcia and C. E. Siewert, A spherical harmonics solution for radiative transfer problems with reflecting boundaries and internal sources, J. Quant. Spectvosc. Radiat. Transfer, 60(2), 1998, 247-260.

[7] C. E. Siewert and C. Devaus, The effect of forward and backward scattering on the law of darkening for the Milne problem and the spherical albedo, J. Quant. Spectrosc. Radiat. Transfer, 20(1), 1978, 29-33.

[8] A. Shafiq, H. E. De Meyer and C. C. Grosjean, The albedo problem in the case of multiple synthetic scattering taking place in a plane- symmetric slab - I and II, Ann. Nucl. Energy, 12(1), 1985, 9-34.

[9] M. C. Gülecyüz and A. Kaskas, The Milne and the constant source problems for the FBIS kernel, Tr. J. of Phys., 22(6), 1998, 461468.

[10] C. Yildiz, Variation of the albedo and the transmission factor with forward and backward scattering in neutron transport theory the $\mathrm{F}_{\mathrm{N}}$ method, Ann. Nucl. Energy, 27(9), 2000, 831-840.

[11] C. Tezcan, M. C. Gülecyüz, R. G. Türeci and A. Kaskas, The $\mathrm{H}_{\mathrm{N}}$ method for half-space albedo and constant source problems for isotropic and anisotropic scattering kernels, J. Quant. Spectrosc. Radiat. Transfer, 103(3), 2007, 611-619.

[12] C. Tezcan and R. Sever, The critical slab with the backward-forward - isotropic scattering model, Ann. Nucl. Energy, 12(10), 1985, 573-576.

[13] C. Tezcan, The Milne problem for isotropic scattering with a backward leak, Transport Theory \& Stat. Phys., 6(2\&3), 1977, 91-99

[14] E.Inönü, A theorem on anisotropic scattering, Transport Theory \& Stat. Phys. 3(2\&3), 1973, $137-146$.

[15] M. H. Haggag, A. R. Degheidy and A. El-Depsy, Efficient and accurate method for radiation transfer problems, J. Quant. Spectrosc. Radiate. Transfer 58(1), 1997, 19-32.

[16] H. F. Machali, A. R. Degheidy and A. El-Depsy, Accurate analytic approximation to Milne's problem for a scattering and absorbing medium, J. Quant. Spectrosc. Radiate. Transfer, 63(1), 1999, 115-125.

[17] M. H. Haggag, A. El-Depsy and D. A. Gharbiea, Two-region Milne problem with anisotropic scattering,Ann. Nucl. Energy, 35(9), 2008, 1701-1707. 